\title{
Amyotrophic Lateral Sclerosis (ALS) about 2 Observations in the Neurology Department of the University Hospital of Cocody
}

\author{
Constance Yapo-Ehounoud1*, Evelyne Aka-Anghui-Diarra1, Muriel Amon-Tanoh1, \\ Berthe Assi' ${ }^{1}$, Ange-Eric Kouamé-Assouan ${ }^{1}$, Christian Tanoh1, Elisée Bony Kotchi ${ }^{2}$ \\ ${ }^{1}$ Neurology Department, University Hospital of Cocody, Abidjan, Ivory Coast \\ ${ }^{2}$ Neurology Department, University Hospital of Bouaké, Bouaké, Ivory Coast \\ Email: ${ }^{*}$ constanceyapoehounoud@gmail.com
}

Received 30 September 2015; accepted 15 October 2015; published 20 October 2015

Copyright (C) 2015 by authors and OALib.

This work is licensed under the Creative Commons Attribution International License (CC BY).

http://creativecommons.org/licenses/by/4.0/

(c) (i) Open Access

\section{Abstract}

Amyotrophic lateral sclerosis (ALS) is a rare but serious neurodegenerative disease characterized by progressive muscular paralysis without overall achievement of higher functions, resulting from motor neuron destruction. The present study reports two cases of elderly female patients aged 45 and 54 years, respectively, allowed for a progressive motor deficit of 4 members. Neurological examination found a spastic pyramidal syndrome and a neurogenic syndrome device of $4 \mathrm{mem}$ bers without sensory syndrome. The ENMG revealed a pure motor neurogenic compatible with involvement of the anterior horn of the spinal cord, holding the ALS diagnosis. The standard radiography and cervical MRI were normal. These patients received analgesics, nursing, physiotherapy and psychotherapy. Then they were lost. ALS is a rare disease (incidence: $1.25 \%$ ). Our patients, with a mean age of 54.5 years, showed clinical and laboratory signs associated with them classically encountered. The diagnosis is suspected in a spastic pyramidal syndrome and neurogenic peripheral syndrome of 4 members but not eliminate cervical spondylotic myelopathy one. The ENMG confirms it. Although the treatment is disappointing, it's based on the riluzole and palliative care, but the evolution is inexorably toward death.

\section{Keywords}

Diagnosis, ALS, Support

Subject Areas: Neurology

\footnotetext{
${ }^{*}$ Corresponding author.
}

How to cite this paper: Yapo-Ehounoud, C., Aka-Anghui-Diarra, E., Amon-Tanoh, M., Assi, B., Kouamé-Assouan, A.-E., Tanoh, C. and Kotchi, E.B. (2015) Amyotrophic Lateral Sclerosis (ALS) about 2 Observations in the Neurology Department of the University Hospital of Cocody. Open Access Library Journal, 2: e1924. http://dx.doi.org/10.4236/oalib.1101924 


\section{Introduction}

Amyotrophic lateral sclerosis (ALS) or Charcot's disease or Lou Gehrig's disease is a neurodegenerative disease characterized by a weakening and paralysis of the legs and arm muscles, breathing muscles, and the muscles of swallowing and speech. Intellectual and sensory functions are not affected. This is a serious progressive disease resulting from neuronal destruction. It reduces the patients' life expectancy.

The prevalence of this disease is estimated at 1/25,000 inhabitants/year. In France, more than 800 new cases are diagnosed each year. It affects slightly more men than women and usually develops between 50 and 70 years. The ALS's pathophysiology is incompletely understood.

The existences of familial forms are $5 \%$ to $10 \%$ of cases possible to achieve animal models that are evaluated neuroprotective molecules potential before passing to humans. Clinically, evolution is marked by extensive progressive paralysis leading to death in variable delays between 2 and 5 years. The progressive profile is heterogeneous and can schematically determine three different profiles: the decliners rapid patients (approximately 25\%); the median survival is less than 12 months of decliners slow patients with disease duration over 5 years $(25 \%)$ or $10(10 \%)$ and patients with a profile classic but with a heterogeneous medium term evolution of 36 months. This evolutionary variability does not allow in clinical practice to predict long-term prognosis although prognostic factors are well identified.

Some regions, including New Guinea and Japan, are characterized by a high prevalence of ALS. In Africa, particularly Ivory Coast, few studies have been done on this subject and ALS is rare, and therefore remains a diagnosis of elimination.

We report the difficulties encountered in 2 patients with Charcot's disease and received in our department over a period of 2 years.

\section{Clinical Case 1}

Young woman 45 years and multipare, Malinké, no occupation, without special history domiciled in Abobo, admitted to service the 27/09/2012 for progressive motor deficit of 4 members evolving for a year in which the interrogation and Clinical examination revealed a spastic tetraparesis of 4 members listed at 0/5 of upper limbs to $3 / 5$ of lower limbs, bilateral thenar atrophy with a aspect of hand of spinal muscular appearance, some fasciculations of the tongue and a diffuse back pain. There are no objective sensory disorders. So we found a cervical myelopathy of inflammatory origin or probable degenerative.

The standard radiographic cervical and cervical CT were normal (see Figure 1). There were no inflammatory syndrome (CBC, SR, CRP normal). The study of CSF showed clear liquid, spring water, zero cytology, and normoglycorachique lymphocyte and normoproteinorachique with no other abnormalities. The ENMG practiced highlighted pure motor neurogenic diffuse supports diffuse anterior horn of spinal cord by holding the diagnosis of ALS in its spinal form. It received symptomatic treatment with analgesic, nursing, physiotherapy and psychotherapy sessions. The administration of riluzole has been proposed, but could not be effective because of its limited accessibility by its high cost.

The patient was medical discharged after two weeks of hospitalization and reviewed 15 days later for another new neurological signs without constipation. She was then definitively lost sight until this day.

\section{Clinical Case 2}

Female patient of 64 years, Senoufo, multipare, housewife, from Korhogo, with notion of inbreeding in his family, was admitted on 08/12/2013 for a progressive motor deficit of 4 members evolving for a year.

Clinical examination revealed a flaccid-spastic tetraparesis of 4 members quoted on $1 / 5$ to $0 / 5$ of the upper limbs and lower limbs, bilateral thenar atrophy with a hand aspect spinal muscular, fasciculations of the tongue, impairment of the IX and X, laughter and tears spasmodic episodes; a foot deformity in varus equine bilateral. There are no objective sensory disorders. So we found a cervical myelopathy of inflammatory origin or probable degenerative.

The cervical MRI was normal (see Figure 2). There were no inflammatory syndrome (CBC, SR, CRP normal). The study of CSF showed clear, spring water, zero cytology, and normoglycorachique normoproteinorachique lymphocytic with no other abnormalities. The ENMG practiced revealed truncal axonal loss of 4 members, distal-proximal no sign of muscle denervation, making finding of ALS in its bulbar and pseudo-polyneuro- 
pathique. She also received symptomatic treatment with analgesic, nursing, physiotherapy and psychotherapy sessions. Treatment with riluzole also met a brake in its case, in view of its cost and its limited availability. The female patient was released from hospital after two weeks at the request of the family. She was then definitively lost sight until this day.

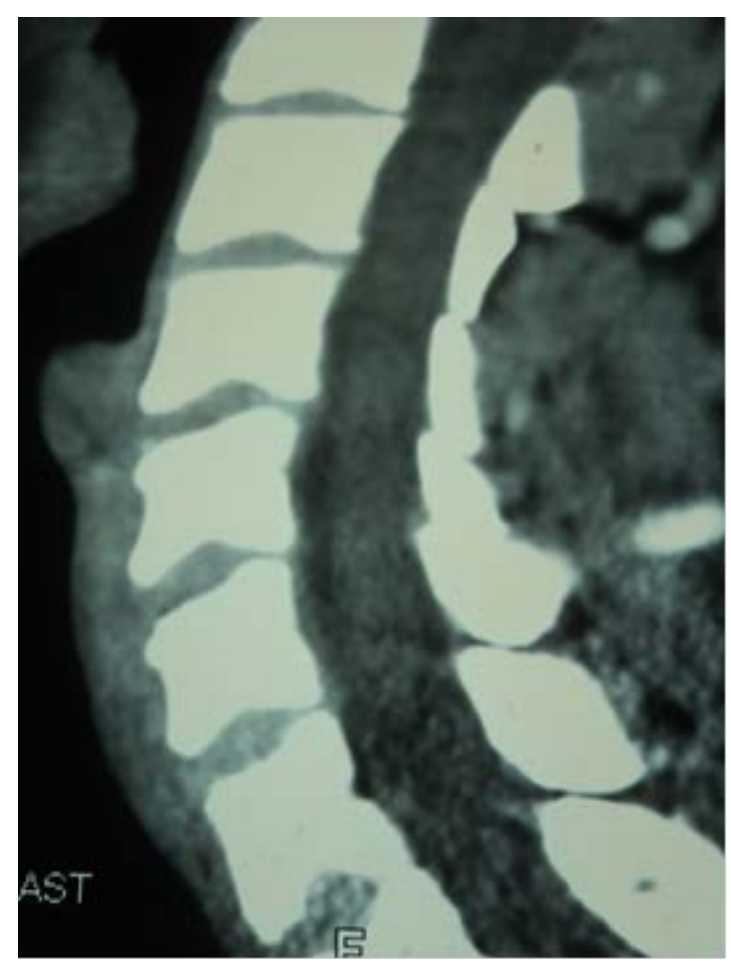

Figure 1. Sagittal section of brain CT scan (Patient 1).

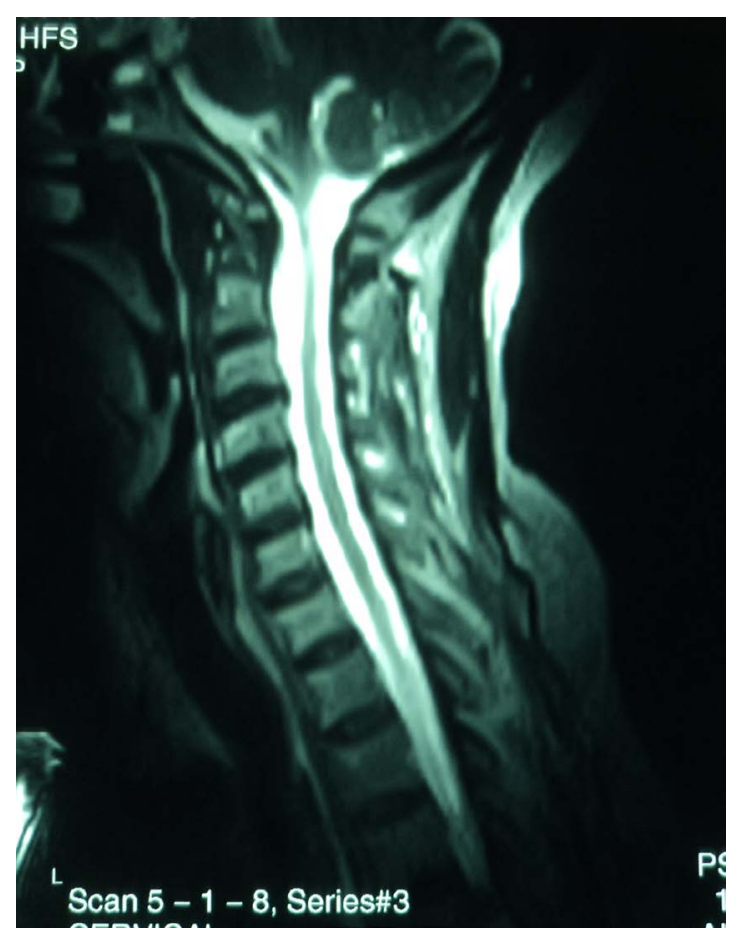

Figure 2. Sagittal section of brain MRI (Patient 2). 


\section{Discussion}

\subsection{Sociodemographics Data}

\subsubsection{Incidence, Age, Sex}

ALS is a rare disease with an incidence of $1.25 \%$, which already represents a small proportion of all patients hospitalized in Neurology and an average age of 54.5 years, which is consistent with the observed age. Our results are similar to those described in the literature [1] [2]. The incidence of ALS appears relatively stable in Caucasian populations of Europe and North America where it is between 1.5 and 2.5/100,000 person-years [3]. The registers of population based on the identification of cases of multiple sources have also contributed to the improvement of the description of the epidemiological profile of the disease [3]. Epidemiological studies performed outside these areas usually show a lower incidence, which is the case in our study. Besides possible differences of susceptibility linked to ethnic origin or possible differences in exposure to exogenous factors, epidemiological methods employed could explain these results [3]. The incidence of disease is very low below 40 years, then increases rapidly after this age to reach a peak incidence between 65 and 75 years [4]. The sex ratio described in the most recent studies is in favor of a slight male predominance (1.5/1) [4]. Our work unlike to those observed have reported a female predominance. Bresch et al. have reported a sex ratio of 0.91 in favor of men, with a mean age of 69 years.

\subsubsection{Prevalence}

Conditioned by the incidence of the disease and the survival time of patients, the prevalence of ALS varies among studies between 3.3 and 7.9/100,000. We have not been able to establish prevalence due to the loss of sight of our two patients.

\subsection{Diagnosis}

\subsubsection{Clinic}

These two clinical cases have helped raise the intricacy of associated genetic and environmental factors occurring in young adult women. Individual and environmental sensitivities have also been highlighted in other studies [4]. The study of the influence of ethnicity and genetic heritage on the occurrence of ALS is growing interest [4]. About the link between ethnicity and survival, the published results remain contradictory. Lee et al. identified in the United States a different evolution according to ethnicity, with a median survival of 18.8 months for "White" subjects, 21.2 months for Hispanics and 49.8 months for "Black" [4]. A study performed in England has not demonstrated difference in survival between Black and White, 38 months vs. 34 months [4]. Other work has identified a shorter survival for non-Whites [4] subjects or from North Africa or the Balkans [4] subjects compared to Caucasians. However, these studies are limited by the tools used (procedures for determining the ethnic origins, classification of subjects Whites/Blacks) and the possibility of differential access to ethnic groups to cares; this allows seeing the great difficulty of access to care in our countries and therefore the actual underestimation of such diseases. The installation method was progressive in all cases over 1 year. Our two patients were Ivorian, from the north of the country, housewives, multiparous and they are mothers at least 5 children. One of them had consanguineous relationships in his family. We deduce that there may be environmental and genetic factors that may explain the onset of this neurodegenerative disease. However no family history of ALS has been reported. In their work, Bresch et al. have noted 3 cases with a family history of ALS, which could explain the risk for these to present a SLA.

Our 2 female patients had spastic tetraparesis, deformation of the hands, fasciculations and a lack of objective sensory disorders as typically encountered in the literature [5].

\subsubsection{Paraclinic}

The ENMG was for a pure motor peripheral neurogenic reached in both cases on a breach of the anterior horn of the spinal cord. The defined ALS diagnosis has been established according to the El Escorial criteria for both patients after clinical examination and EMG. Other authors have reported the importance of these criteria in the diagnosis of ALS [6]. This diagnosis is mainly based on neurological examination and electro-neuro-myogram (ENMG). It is easy in conventional forms involving damage of the peripheral motor neuron (NMP) and central motor neuron (NMC), of progressive evolution and touching multiple territories. It may be more difficult in the 
beginning of the disease, or before to certain clinical presentations. The delay in diagnosis varies from 7 to 12 months depending on the time and remains a neurological expertise [7]. This delay was also noticed for our female patients, who have benefited from a neurological consultation until 12 months after installation of signs.

The electrophysiological diagnosis can sometimes be completed by other examinations including Motor Evoked Potentials (MEP). If imaging techniques can in certain circumstances be an aid to diagnosis by showing damage of the central motor neuron, they mainly involved in the differential diagnosis. The study of cerebrospinal fluid (CSF), examination privileged during the study of the nervous system, has an essential role in the differential diagnosis. It is normal in ALS: there is neither cellular reaction nor elevated protein, which has been reported in our two cases. The presence of an abnormality is therefore a guidance element towards another disease: elevated protein evokes a spinal cord compression, a paraneoplastic syndrome (association with a lymphoma or a cancer); cell response directed towards an infectious process (Lyme disease, syphilis, HIV), a lymphoma or neoplastic process (abnormal cells) [8].

\subsubsection{Supported}

The treatment was based on symptomatic measures coupled with physiotherapy and psychotherapy. Our main clash was the administration of riluzole, the cost was a real brake on the support of these patients because expensive for them. Yet therapeutic interventions with riluzole type prescription [9], gastrostomy and noninvasive ventilation [9] have shown their interest in improving the survival of ALS patients [9].

\subsubsection{Evolution and Prognosis}

The 2 female patients were all lost of sight after two weeks of hospitalization, in contrast to what is usually described [10]-[12]. This can show the unknown feature of this disease and the mystical aspect that is sometimes vested in the northern regions of our country. Furthermore it was also a bias in the subsequent monitoring especially as one of them already had a bulbar shape, so likely to cause swallowing and respiratory disorders or respiratory arrest.

\section{Conclusion}

ALS or Lou Gehrig's disease remains common in our hospital practice. It remains a rare disease and an elimination diagnosis before mainly the cervical spondylitis, the polyradiculoneuropathies (PRN) and infectious meningomyeloradiculites dominated by spinal tuberculosis in our current practice. Therefore, its support should involve a multidisciplinary approach (neurologist, physical therapist, psychologist, religious, and family environment) and require palliative care. The commonly used medical treatment remains inaccessible to our population because it is too expensive for them.

\section{Declaration of Interests}

The authors declare that they have no conflicts of interest related to this article.

The local ethic committee and the patients have given their agree to made this paper.

\section{References}

[1] (2008) General Public Orphanet Encyclopedia. Amyotrophic Lateral Sclerosis. Mai. https://www.orpha.net/data/patho/Pub/fr/ScleroseLateraleAmyotrophique-FRfrPub106.pdf/

[2] Leif Sigurdson, A. (2011) Amyotrophic Lateral Sclerosis Presenting as Upper Limb Weakness in a 35 Years Old Female: A Case Report. Journal of the Canadian Chiropractic Association, 55, 204-210.

[3] Marin, P.C.B., Nicol, G.L.M. and Preux, P.-M. (2014) Epidemiology, Clinical Spectrum of ALS and Differential Diagnoses. La Presse Médicale, 43, 538-548. www.em-consulte.com/revue/lpm www.sciencedirect.com

[4] Lee, J.R., Annegers, J.F. and Call, S.H. (1995) Prognosis of Amyotrophic Lateral Sclerosis and the Effect of Referral Selection. Journal of the Neurological Sciences, 132, 207-215. http://dx.doi.org/10.1016/0022-510X(95)00154-T

[5] Fischer, L.R., et al. (2004) Amyotrophic Lateral Sclerosis Is a Distal Axonopathy: Evidence in Mice and Man. Experimental Neurology, 185, 232-240. http://dx.doi.org/10.1016/j.expneurol.2003.10.004

[6] Bresch, S., Delmont, E., Soriani, M.H. and Desnuelle, C. (2014) Contribution of Electromyography in the Early Diagnosis of ALS to Bulbar Onset: Comparison of El Escorial Criteria, Modified El Escorial and Awaji. Neurological Re- 
view, 170, 134-139. www.sciencedirect.com

[7] Traynor, B.J., et al. (2000) Clinical Features of Amyotrophic Lateral Sclerosis Selon El Escorial and the Airlie House Diagnostic Criteria: A Population Based Study. Archives of Neurology, 57, 1171-1176. http://dx.doi.org/10.1001/archneur.57.8.1171

[8] Juliette, S., Choumert, A., Vandenberghe, N., Gervais, H.B., Bouhour, F. and Vial, E.B.C. (2003) Diagnosis of Amyotrophic Lateral Sclerosis (ALS) through Excess: Experience of ALS Center of Lyon about 18 Patients. Summary of JNLF Montpellier.

[9] Bensimon, G., et al. (1994) A Controlled Trial of Riluzole in Amyotrophic Lateral Sclerosis. ALS/Riluzole Study Group. New England Journal of Medicine, 330, 585-591. http://dx.doi.org/10.1056/NEJM199403033300901

[10] Pradat, P.F., et al. (2009) News in Basic and Clinical Research on ALS. Revue Neurologique (Paris).

[11] Pringle, E.C., et al. (1992) Primary Lateral Sclerosis. Clinical Features, Neuropathology and Diagnostic Criteria. Brain, 115, 495-520. http://dx.doi.org/10.1093/brain/115.2.495

[12] Pradat, P.F., et al. (2006) What Are the Differential Diagnoses and Forms Borders of ALS? Revue Neurologique (Paris), 162, S81-S90. http://dx.doi.org/10.1016/S0035-3787(06)75168-2

\section{Glossary}

ALS: amyotrophic lateral sclerosis;

CBC: cells blood count;

CRP: C-reactive protein;

ENMG: electroneuromyogram;

SR: sedimentation rate. 